Virginia Commonwealth University VCU Scholars Compass

Physics Publications

Dept. of Physics

2010

\title{
Surface photovoltage in undoped n-type GaN
}

M.A. Reshchikov

Virginia Commonwealth University, mreshchikov@vcu.edu

M. Foussekis

Virginia Commonwealth University

A. A. Baski

Virginia Commonwealth University, aabaski@vcu.edu

Follow this and additional works at: http://scholarscompass.vcu.edu/phys_pubs

Part of the Physics Commons

Reshchikov, M. A., Foussekis, M., \& Baski, A. A. Surface photovoltage in undoped n-type GaN. Journal of Applied Physics, 107, 113535 (2010). Copyright (C) 2010 American Institute of Physics.

\section{Downloaded from}

http://scholarscompass.vcu.edu/phys_pubs/123

This Article is brought to you for free and open access by the Dept. of Physics at VCU Scholars Compass. It has been accepted for inclusion in Physics Publications by an authorized administrator of VCU Scholars Compass. For more information, please contact libcompass@vcu.edu. 


\title{
Surface photovoltage in undoped $n$-type GaN
}

\author{
M. A. Reshchikov, ${ }^{\text {a) }}$ M. Foussekis, and A. A. Baski \\ Department of Physics, Virginia Commonwealth University, Richmond, Virginia 23284, USA
}

(Received 17 March 2010; accepted 6 April 2010; published online 11 June 2010)

\begin{abstract}
Steady-state and transient surface photovoltage (SPV) in undoped GaN is studied in vacuum and air ambient at room temperature and $400 \mathrm{~K}$ with a Kelvin probe. The results are explained within a phenomenological model accounting for the accumulation of photogenerated holes at the surface, capture of free electrons from the bulk over the near-surface potential barrier, and emission of electrons from surface states into the bulk. Simple analytical expressions are obtained and compared with experimental results. In particular, the proposed model explains the logarithmic decay of the SPV after stopping illumination. Internal and external mechanisms of the SPV are discussed in detail. It is shown that an internal mechanism dominates at low illumination intensity and/or small photon energies, while external mechanisms such as charging of a surface oxide layer and photoinduced processes play a significant role for above-bandgap illumination with sufficient intensity. (C) 2010 American Institute of Physics. [doi:10.1063/1.3430979]
\end{abstract}

\section{INTRODUCTION}

Progress in the development of GaN-based lightemitting and electronic devices contrasts with a weak understanding of the bulk and surface defects that play a pivotal role in the quantum efficiency and reliability of optical and electronic devices. Surfaces of air-exposed, undoped $n$-type GaN grown on $c$-plane sapphire show an upward band bending of roughly $1 \mathrm{eV}$ due to negative charge at the surface ${ }^{1-4}$ but the source of this charge is not well established. As in other semiconductors, this negative charge may be associated with intrinsic surface states due to dangling bonds, ${ }^{5}$ impurities, ${ }^{6}$ native defects, ${ }^{7}$ surface reconstructions, random stress, ${ }^{8}$ etc. It may also be related to extrinsic sources such as atoms or molecules adsorbed from the air or defect states in a thin surface oxide layer. It is known that about a monolayer of chemisorbed oxygen covers the GaN surface, ${ }^{1,9}$ and a thin $(\sim 1 \mathrm{~nm})$ oxide layer may also be formed. ${ }^{10}$ Illumination of $\mathrm{GaN}$ with ultraviolet (UV) light can reduce the surface band bending by $0.3-1.1 \mathrm{eV}$ due to the accumulation of photogenerated holes at the surface, i.e., the surface photovoltage (SPV) effect. ${ }^{11-14}$ After pulsed illumination, the SPV signal in GaN decays very slowly as a logarithmic function of time. $^{13}$ This behavior is similar to the SPV decay in GaAs (Refs. 15 and 16) but orders of magnitude slower than in $\mathrm{Si}^{17}$

Several phenomenological models have been proposed to quantitatively explain the SPV in semiconductors. ${ }^{18-29}$ Some of these models are general and necessarily complex so that the solutions can only be obtained by using numerical or graphical methods. ${ }^{21,22,27,29}$ Other models are limited in scope. In particular, Lagowski et al. ${ }^{21,23}$ investigated the SPV arising in semiconductors due to resonant below-bandgap excitation of surface states arising from adsorbed species. Further, there appear to be no simple explanations for the logarithmic decay of the SPV after stopping illumination, except

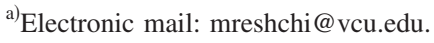

in the case of a very small SPV. ${ }^{13}$ Note that some models of SPV transients assume the decay to be exponential, which has led to confusion in the literature. ${ }^{30,31}$

Often, it is assumed in modeling that the carrier diffusion length considerably exceeds the depletion region width, ${ }^{22,29}$ and the SPV technique is commonly regarded as a method for measuring the minority carrier diffusion length. ${ }^{27,28,32}$ However, this is not the case for GaN layers grown by metalorganic chemical vapor deposition (MOCVD) and molecular beam epitaxy (MBE) on sapphire substrates. A high density of threading dislocations $\left(10^{9}-10^{10} \mathrm{~cm}^{-2}\right)$ results in a very small minority carrier diffusion length of $0.02-0.3 \mu \mathrm{m},{ }^{33-36}$ smaller than the typical depletion region width in undoped GaN samples. Here, we develop a phenomenological model with analytical expressions for steady-state SPV and its transients that can be used for undoped $\mathrm{GaN}$ and other semiconductors with short diffusion lengths.

Another goal of this work is to elucidate the roles of internal and external mechanisms in SPV measurements. It was noted in early studies that two different types of surface states, called slow and fast, contribute to surface band bending and SPV in semiconductors. ${ }^{29,37,38}$ The fast states are located at the semiconductor-oxide interface and are believed to be independent of ambient changes. The slow states are located on or within the surface oxide layer and are affected by the ambient atmosphere. Often, the charge due to adsorbed ionized species is considered to be fixed ${ }^{22}$ and does not affect the SPV, at least under low-intensity illumination that does not lead to photochemical processes such as photodesorption or photoadsorption. ${ }^{18}$ However, charge transfer between adsorbed species and the semiconductor has also been considered as the principle mechanism of SPV. ${ }^{39}$ At present, specific mechanisms of SPV in GaN, the role of adsorbed species and "internal" surface states in electrical and optical properties, and the surface state energy distribution in $n$-type GaN remain largely unknown. By comparing SPV and photoluminescence data, Shalish et al. ${ }^{14,40}$ sug- 


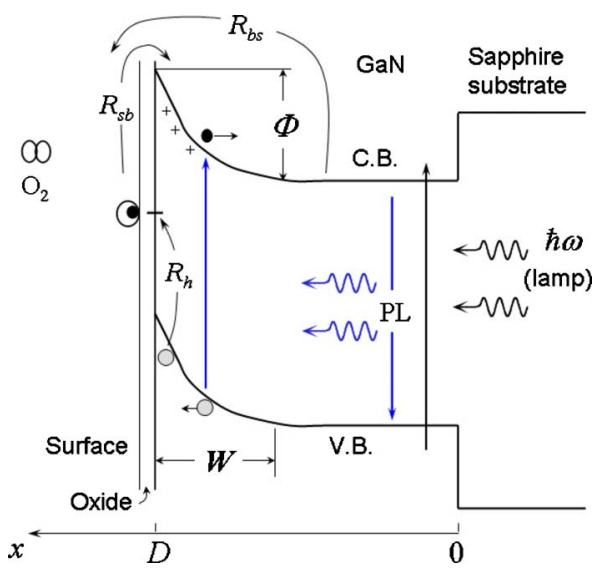

FIG. 1. (Color online) Schematic band diagram and main transitions in the studied samples. Direction of illumination and the $x$ axis correspond to configuration A. In configuration B, illumination is from the left and the $x$ axis is directed to the right with zero at the $\mathrm{GaN}$ surface.

gested that the upward band bending in $n$-type $\mathrm{GaN}$ is caused by negatively charged surface acceptors located $\sim 2.2 \mathrm{eV}$ below the conduction band. They also attributed the notorious yellow luminescence band to these acceptors. It should be noted, however, that the high sensitivity of photoluminescence in $\mathrm{GaN}$ to the ambient implies that adsorbed species play a significant role in band bending. ${ }^{41,42}$ Our analysis of the SPV in undoped GaN allows us to distinguish internal and external sources of surface charge and their contributions to near-surface carrier transport.

In the present work, we have studied steady-state and transient photovoltage in $n$-type GaN under vacuum and ambient conditions at different temperatures, and have developed a phenomenological model with simple analytical expressions which explains most of the observed effects. This paper comprises the following sections: theoretical background where expressions are derived for steady-state and transient SPV for below-bandgap and above-bandgap excitation in $\mathrm{GaN}$ (Sec. II), experimental details for Kelvin probe measurements (Sec. III), experimental SPV results for GaN and comparison to theory (Secs. IV and V), and main conclusions (Sec. VI).

\section{THEORY}

\section{A. Photovoltage caused by light absorption in the depletion region and at the surface}

A band diagram for $n$-type GaN grown on sapphire with a thin surface oxide film is shown schematically in Fig. 1. At the surface of $n$-type $\mathrm{GaN}$, negative charge with density $n_{s}$ causes upward band bending characterized by a barrier height $\Phi$ and depletion region width $W$ given by ${ }^{24}$

$$
W=\frac{n_{s}}{N_{D}}=\sqrt{\frac{2 \Phi \epsilon \epsilon_{0}}{q^{2} N_{D}}},
$$

where $\epsilon$ is the static dielectric constant $[\epsilon=9.8$ for GaN (Ref. 43)], $\epsilon_{0}$ is the vacuum permittivity, $q$ is the electron charge, and $N_{D}$ is the concentration of uncompensated shallow donors. Illumination reduces the band bending by the SPV value $y=\Phi_{0}-\Phi$, where $\Phi_{0}$ is the initial barrier height under dark conditions. Although light-induced transitions in the bulk and especially in the depletion region may contribute to the photovoltage signal, ${ }^{18,44}$ we will assume to first approximation that the SPV is primarily caused by a variation in the negative charge density $n_{s}$ at the semiconductor surface expressed as

$$
n_{s}(y)=\sqrt{\frac{2\left(\Phi_{0}-y\right) \epsilon \epsilon_{0} N_{D}}{q^{2}}}=n_{s}(0) \sqrt{1-\frac{y}{\Phi_{0}}},
$$

where $n_{s}(0)$ is the initial density of charged surface states occurring under dark conditions.

Two sources of the negative surface charge for $n$-type semiconductors have been suggested as follows: (1) species from the ambient that are adsorbed at the surface and (2) semiconductor or interface states that are formed due to disorder, dangling bonds, point defects, etc. In the first case, the charge is often considered to be fixed and the exchange of electrons between the adsorbed species and semiconductor is expected to be very slow. In the second case, photogenerated holes can quickly change the surface charge, and transitions via the surface states are commonly expected to be fast. Note, however, that classification of surface states into slow and fast and charges into fixed and variable should be done with care. Indeed, transitions of electrons from the bulk to surface states over a high near-surface barrier may be very slow. On the other hand, "fixed" charge may change in time due to photoinduced adsorption or desorption. The internal and external mechanisms of charging are now considered in some detail below.

In the internal mechanism of SPV it is assumed that states with a total density $N_{s}$ (including all acceptors and donors with densities of $N_{s A}$ and $N_{s D}$, respectively) are located at the semiconductor surface, of which $n_{s}$ is the net density of negatively charged states. Note, that negatively charged acceptors may be partially compensated by positively charged donors, i.e., $n_{s}=N_{s A}^{-}-N_{s D}^{+}$. Both continuous and discrete distributions of surface states are expected; however, their energy distributions do not affect the main conclusions of this work, as will be shown later. The capture of bulk electrons by unoccupied surface states (with density $N_{s}^{*}=N_{s A}-n_{s}$ ) and the emission of electrons from occupied surface states (with density $n_{s}^{*}=n_{s}+N_{s D}$ ) to the bulk are described by the effective electron-capture coefficients $C_{n 1}$ and $C_{n 2}$, respectively. In the case of several discrete surface states, these phenomenological parameters would represent average values. Note that $N_{s A}, N_{s D}, N_{s}^{*}$, and $n_{s}^{*}$ are of the order of $10^{14} \mathrm{~cm}^{-2}$, whereas $n_{s}$ is of the order $10^{12} \mathrm{~cm}^{-2}$. The consideration of charged and uncharged donors and acceptors in our model makes it more realistic in comparison to some other models where the total density of surface states is too small. ${ }^{21}$

In the external mechanism of SPV it is assumed ${ }^{39}$ that physisorbed oxygen or other species form unoccupied states at the surface with density $N_{s}^{*}$ which can accept electrons from the bulk and become chemisorbed. The density of chemisorbed or negatively charged states is $n_{s}$. The reverse process would be the conversion of chemisorbed species into physisorbed ones by either capturing a hole from bulk or exciting an electron to the conduction band. In a simple phe- 
nomenological approach, the ability of the unoccupied (physisorbed) states to capture electrons will be characterized by the electron-capture coefficient $C_{n 1}$, and the ability of the occupied (chemisorbed) states to give up the electrons will be characterized by the coefficient $C_{n 2}$. The same notation can be used to describe the capture coefficients for both the external and internal charging mechanisms because they can be formally described in a similar way. It should be noted, however, that the effective electron capture cross-sections for the external mechanism may be much smaller and the capture and emission rates much slower than for the internal mechanism because processes such as electron tunneling and relatively slow chemical reactions may be involved in the former case.

In general, the SPV for an $n$-type semiconductor may result from photons being absorbed at semiconductor surface states, in the depletion region, or in the bulk (Dember potential $\left.^{45}\right)$. In the case of a bulk contribution, the Dember potential may arise due to the nonuniform generation of electrons and holes and their subsequent diffusion with different mobilities. For the GaN samples used in this study, this bulk contribution to the SPV can be ignored because the excess minority carrier concentration does not exceed the equilibrium electron concentration. Moreover, the Dember potential should have an opposite sign for front-side versus backside illumination. We observed, however, that the SPV signal was about the same for both geometries using band-to-band excitation at the highest illumination intensity. Therefore, our analysis of the SPV only includes the resonant excitation of surface states (dominant mechanism for photon energies below bandgap) and photon absorption in the depletion region (dominant mechanism for photon energies equal to or greater than the bandgap).

In our experiments, the samples are illuminated from the backside through a sapphire substrate (Fig. 1, configuration A). The light intensity $P_{0}$, therefore, decreases inside the GaN layer as $\exp (-\alpha x)$, where $\alpha$ is the absorption coefficient and $x=0$ at the $\mathrm{GaN} /$ sapphire interface and $x=D$ at the $\mathrm{GaN}$ surface. The light fluxes (photons per square centimeter per second) absorbed in the depletion layer $P_{W}$ and at the surface states $P_{S}$ are

$$
P_{W}=\int_{D-W}^{D} \alpha P_{0} e^{-\alpha x} d x=P_{0} e^{-\alpha D}\left(e^{\alpha W}-1\right)
$$

and

$$
P_{S}=\sigma_{p h}^{s} n_{s}^{*} P_{0} e^{-\alpha D},
$$

where $\sigma_{p h}^{s}=\sigma_{p h}^{s}(\hbar \omega)$ is by definition ${ }^{18}$ the optical crosssection of the surface states for photons with energy $\hbar \omega$. In the latter case, photons have sufficient energy to excite electrons from the surface states into the conduction band. Such resonant excitation may or may not be followed by conversion of chemisorbed species to physisorbed ones.

When the front side of the sample is illuminated with above-bandgap light (Fig. 1, configuration B with $x=0$ at the GaN surface), absorption of photons in the bulk can be neglected to yield

$$
P_{W}=\int_{0}^{W} \alpha P_{0} e^{-\alpha x} d x=P_{0}\left(1-e^{-\alpha W}\right)
$$

and

$$
P_{S}=\sigma_{p h}^{s} n_{s}^{*} P_{0} .
$$

For the common case of $W \ll D, P_{W}$ has a maximum in configuration $\mathrm{A}$ when $\alpha D \approx 1$ and in configuration $\mathrm{B}$ when $\alpha W \gg 1$. For example, in a $2.5 \mu \mathrm{m}$-thick $\mathrm{GaN}$ layer with a $0.15 \mu \mathrm{m}$-wide depletion region, the maximum amount of light absorbed in the depletion region is $2 \%$ in configuration A when $\alpha \approx 4 \times 10^{3} \mathrm{~cm}^{-1}$ (or at photon energies close to $\mathrm{GaN}$ bandgap), and more than $80 \%$ in configuration $\mathrm{B}$ when $\alpha>10^{5} \mathrm{~cm}^{-1}$ (or at photon energies above GaN bandgap). Note, that due to a strong electric field in the depletion region, photons with an energy slightly less than the bandgap will be effectively absorbed in the depletion region via photon-assisted tunneling (Franz-Keldysh effect), thereby increasing absorption in the depletion region.

Illumination with photons having an energy close to or above the bandgap creates electron-hole pairs in the depletion region that are quickly separated by the strong electric field. To first approximation all of the holes reach the surface and recombine with surface electrons (located at semiconductor surface states for internal mechanism or at adsorbed species for external mechanism), thus reducing the band bending. We will neglect the diffusion of holes into the bulk because in the undoped GaN layers studied here with a threading dislocation density of mid $10^{9} \mathrm{~cm}^{-2}$, the diffusion length is expected to be equal to or smaller than the depletion region width. Moreover, the strong electric field near the surface $\left(\sim 6 \times 10^{4} \mathrm{~V} / \mathrm{cm}\right)$ quickly sweeps photogenerated holes to the surface. Consequently, the rate at which holes flow to the surface in the case of band-to-band excitation is equal to $P_{W}$. Photons having an energy lower than the bandgap, for which $\alpha D \ll 1$, easily pass through the GaN layer in any configuration. They can resonantly excite electrons at surface states to overcome the surface barrier and be swept into the bulk by the electric field, thus also reducing the band bending. The rate of this process is equal to $P_{S}$.

In principle, photons with below-bandgap energy can also be absorbed by deep-level defects in the depletion region and contribute to the SPV signal. A number of electron and hole traps with concentrations around $10^{15} \mathrm{~cm}^{-3}$ and deep levels from 0.6 to $3.3 \mathrm{eV}$ below the conduction band were reported for $n$-type GaN grown by MBE. ${ }^{46}$ Electrons can be excited from these defect states to the conduction band, followed by the excitation of electrons from the valence band to the empty defect levels. This contribution is commonly ignored. ${ }^{18}$ This can be justified by the low concentration of deep-level defects in the depletion region (as compared to the surface state density), the relatively high concentration of free electrons in equilibrium, and the low probability of two-step excitations at low illumination intensity.

In addition to the light-induced transitions described above, thermionic transitions of electrons occur between the surface states and bulk both with and without illumination, where such transitions obey Boltzmann statistics. Free elec- 
trons from the bulk region can overcome the barrier height $\Phi$ and be trapped by surface states. The rate $R_{b s}$ of this bulkto-surface flow of free electrons is

$$
\begin{aligned}
R_{b s} & =C_{n 1} N_{s}^{*} n \exp \left(-\frac{\Phi_{0}-y}{k T}\right) \\
& =C_{n 1} N_{s}^{*} N_{C} \exp \left(-\frac{\Phi_{0}+\left(E_{C}-F\right)-y}{k T}\right),
\end{aligned}
$$

which can be rewritten as

$$
R_{b s}=R_{0} \exp \left(\frac{y}{k T}\right),
$$

where

$$
R_{0}=C_{n 1} N_{s}^{*} N_{C} \exp \left(-\frac{\Phi_{0}+E_{C}-F}{k T}\right) .
$$

The rate $R_{0}$ is the bulk-to-surface flow of free electrons under initial dark conditions. In these expressions, $C_{n 1}$ is the effective electron-capture coefficient described earlier, $N_{C}$ is the effective density of states in the conduction band, $N_{s}^{*}$ is the density of unoccupied states at the surface, $E_{C}$ and $F$ are the energies of the conduction band edge and the Fermi level in bulk, respectively, $y$ is the SPV value, $k$ is Boltzmann's constant, and $T$ is temperature. With regard to $N_{s}^{*}$, hereafter it will refer to either the density of semiconductor surface states able to trap electrons from the bulk (internal mechanism) or the density of physisorbed species able to accept electrons from the bulk and become chemisorbed (external mechanism). Electrons trapped at the surface can also overcome the barrier and escape to the bulk region. For simplicity, we will consider here a single surface state level with energy $E_{S}$ and will later investigate how the results change if several discrete or continuous surface states are present at the surface. The rate of surface-to-bulk flow $R_{s b}$ of electrons emitted from the surface level is

$$
R_{s b}=C_{n 2} n_{s}^{*} N_{C} \exp \left(-\frac{E_{C}-E_{S}}{k T}\right)
$$

where $n_{s}^{*}$ is the density of surface states occupied with electrons. If the surface state energy level pins the Fermi level and controls the band bending in dark, $E_{C}-E_{S}=\Phi_{0}+\left(E_{C}\right.$ $-F$ ). In the internal mechanism, the coefficients $C_{n 1}$ and $C_{n 2}$ can be expressed via the electron capture cross-sections $\sigma_{n 1}$ and $\sigma_{n 2}$, respectively, as:

$$
C_{n 1,2}=\sigma_{n 1,2} v_{n}=\sigma_{n 1,2} \sqrt{\frac{8 k T}{\pi m_{e}^{*}}},
$$

where $v_{n}$ is the average electron thermal velocity and $m_{e}^{*}$ is the effective electron mass in the conduction band. Note, that the surface electron recombination velocity $s_{n}=C_{n 1} N_{s}^{*}$ is often used instead of the capture coefficient.

Under dark conditions, the flow of free electrons is in equilibrium $\left(R_{b s}=R_{s b}\right)$ and results in the following relation:

$$
C_{n 1} N_{s}^{*}=C_{n 2} n_{s}^{*} \text {. }
$$

Note that $N_{s}^{*}>10^{14} \mathrm{~cm}^{-2}$ for a monolayer of physisorbed oxygen (external mechanism) and for a typical semiconduc- tor surface state density (internal mechanism), while $n_{s}$ $<10^{12} \mathrm{~cm}^{-2}$ with typical parameters of $\Phi_{0} \approx 1 \mathrm{eV}$ and $N_{D}$ $\approx 10^{17} \mathrm{~cm}^{-3}$ for GaN. Since $N_{s}^{*}$ and $n_{s}^{*}$ are much larger than $n_{s}$ in dark and under illumination, Eq. (12) holds at any illumination level. In the presence of light, the rate equation can be written in general as

$$
\frac{d n_{s}}{d t}=R_{b s}-R_{s b}-R^{*},
$$

where $R^{*}$ is the bulk-to-surface flow of light-induced holes due to the separation of electron-hole pairs (above-bandgap excitation) or surface-to-bulk flow of resonantly excited electrons from surface states (below-bandgap excitation). In general, $R^{*}$ can be defined as

$$
R^{*}=c P_{0} .
$$

In the case of band-to-band excitation with backside illumination $\left(R^{*}=P_{W}\right)$, the coefficient $c$ is defined as:

$$
c=e^{-\alpha D}\left(e^{\alpha W}-1\right) \text {. }
$$

In the case of resonant excitation of surface states with either front or backside illumination $\left(R^{*}=P_{S} \approx \sigma_{p h}^{s} n_{s}^{*} P_{0}\right)$, the coefficient is given by

$$
c=\sigma_{p h}^{s} n_{s}^{*} .
$$

Using Eqs. (2), (8), (10), and (12), Eq. (13) takes the following form:

$$
-\frac{n_{s}(0)}{2 \Phi_{0} \sqrt{1-\frac{y}{\Phi_{0}}}} \frac{d y}{d t}=R_{0} \exp \left(\frac{y}{\eta k T}\right)-R_{0}-R^{*} .
$$

For generality, we have introduced a phenomenological factor $\eta$ in the term describing the capture of free electrons by surface states, which has a meaning similar to the ideality factor in Schottky diodes. ${ }^{24}$ But unlike for Schottky diodes where $\eta$ varies between 1 and $2, \eta$ varies between 0.5 and 2 for different models of a free surface. ${ }^{18}$ For example, $\eta<1$ when trapping occurs at the surface and $\eta \approx 2$ when significant trapping occurs in the depletion region. ${ }^{47}$

After illumination begins, the photovoltage $y$ increases from zero to the saturation value $y_{0}$ when the steady-state condition is achieved, i.e., when $d y / d t=0$. After switching off the illumination $\left(R^{*}=0\right)$, the photovoltage gradually decreases from $y=y_{0}$ to zero. We have numerically solved Eq. (17) for all three cases (rise, steady-state condition, and decay of SPV) and have also obtained approximate solutions for the certain-situations presented below.

\section{B. Steady-state photovoltage and its dependence on light intensity}

Under continuous illumination, the net flow of electrons to the surface is equal to the flow of photogenerated holes to the surface, i.e., $R_{b s}-R_{s b}=R^{*}$ in steady-state condition, and Eq. (17) becomes

$$
\exp \left(\frac{y_{0}}{\eta k T}\right)=\frac{R^{*}}{R_{0}}+1 \text {. }
$$


After substitution for $R^{*}$ using Eq. (14), we then obtain the solution for Eq. (18) in the form

$$
y_{0}=\eta k T \ln \left(\frac{c P_{0}}{R_{0}}+1\right) \text {. }
$$

This dependence represents linear and logarithmic increases in photovoltage with light power for $c P_{0}<R_{0}$ and $c P_{0}>R_{0}$, respectively. Equation (19) can be used for a wide range of illumination intensities up to the SPV saturation value, which corresponds to a flattening of the bands. By fitting Eq. (19) to experimental $y_{0}\left(P_{0}\right)$ data obtained with band-to-band and resonant below-bandgap excitation, one can estimate the values of $\sigma_{n 1}$ and $\sigma_{p h}^{s}$, respectively, provided that the value of $\Phi_{0}$ is known.

Equation (19) can be compared to an expression originally derived within thermionic emission theory for current transport in metal-semiconductor contacts ${ }^{24}$ and commonly used in the analysis of photovoltage as a function of light intensity ${ }^{26,30,48}$

$$
y_{0}=\eta k T \ln \left(\frac{I_{p h}}{j_{0}}+1\right) .
$$

Here, $I_{p h}$ is the current of carriers generated by light, $\eta$ is the ideality factor which is close to unity, and $j_{0}$ is the metal-tosemiconductor saturation current in a Schottky diode given by $^{24}$

$$
j_{0}=A^{*} T^{2} \exp \left(-\frac{\Phi_{0}+\left(E_{C}-F\right)}{k T}\right) .
$$

In this expression, $A^{*}$ is the effective Richardson constant for thermionic emission

$$
A^{*}=\frac{4 \pi q m_{e}^{*} k^{2}}{h^{3}}
$$

where $h$ is Plank's constant. The main difference between Eqs. (19) and (20) is a factor of $4 \sigma_{n 1} N_{s}^{*}=4 s_{n}$ that appears before $j_{0}$ after substitutions, which is typically significantly larger than unity.

\section{Photovoltage transients}

When a sample is illuminated after being stored in the dark for a long period of time, the initial slope $d y / d t$ of the photovoltage rise is proportional to $P_{W}$ in the case of abovebandgap excitation for $y \ll \Phi_{0}$, as can be seen from Eq. (17)

$$
\frac{d y}{d t} \approx \frac{2 \Phi_{0} R^{*}}{n_{s}(0)}=\frac{2 \Phi_{0} c P_{0}}{n_{s}(0)}=2 c P_{0} \sqrt{\frac{q^{2} \Phi_{0}}{2 \varepsilon \varepsilon_{0} N_{D}}} .
$$

Thus, the value of $\Phi_{0}$ can be determined from the initial slope of the SPV rise under band-to-band illumination (when $R^{*}=P_{W}$ is known). The following relation between $y$ and $t$, describing the SPV rise starting at $t=0$, can also be obtained from Eq. (17) in the case of $y \ll \Phi_{0}$

$$
t \approx \frac{n_{s}(0)}{2 \Phi_{0}\left(R_{0}+c P_{0}\right)}\left\{y-\eta k T \ln \left[1+\frac{R_{0}}{c P_{0}}\left(1-e^{y / \eta k T}\right)\right]\right\} .
$$

For resonant below-bandgap excitation, the initial slope of the $d y / d t$ dependence gives the optical cross-section for the surface states when $\Phi_{0}$ is known since $R^{*} \approx \sigma_{p h}^{s} n_{s}(0) P_{0}$ and

$$
\frac{d y}{d t} \approx 2 \Phi_{0} \sigma_{p h}^{s} P_{0}
$$

for the resonant excitation of surface states.

When the illumination is switched off $\left(R^{*}=0\right)$ after saturation of the photovoltage signal, the photovoltage decreases from $y=y_{0}$ at $t=t_{1}$ to $y=0$ at $t \rightarrow \infty$. The following relation between $y$ and $t$, describing the decay of SPV starting at $t$ $=t_{1}$, can be obtained from Eq. (17) in the case of $y \ll \Phi_{0}$

$$
t-t_{1} \approx \frac{n_{s}(0)}{2 \Phi_{0} R_{0}}\left[y-y_{0}+\eta k T \ln \left(\frac{e^{y_{0} / \eta k T}-1}{e^{y / \eta k T}-1}\right)\right] .
$$

A simplified analytical expression for $y(t)$ can then be derived from Eq. (26) in the region of photovoltage decay where $k T \ll y \ll \Phi_{0}$ by using the approximations $\sqrt{\Phi_{0}-y}$ $\approx \sqrt{\Phi_{0}}$ and $\exp (y / k T) \gg 1$ to give

$$
y \approx y_{0}-\eta k T \ln \left(1+\frac{t-t_{1}}{\tau}\right),
$$

where

$$
\tau=\tau_{0} \exp \left(-\frac{y_{0}}{\eta k T}\right)
$$

with

$$
\tau_{0}=\frac{\eta k T}{R_{0}} \frac{n_{s}(0)}{2 \Phi_{0}} .
$$

Thus, in some range of $y(t)$ there is a logarithmic dependence with slope $\eta k T$ which depends on temperature but is independent of other parameters. Note, that for $t-t_{1} \gg \tau$, Eq. (27) simplifies to

$$
y \approx-\eta k T \ln \left(\frac{t-t_{1}}{\tau_{0}}\right) .
$$

Figure 2 compares the time dependences of the photovoltage transients obtained by numerical calculations using Eq. (17) with the dependences obtained using the approximate formulas in Eqs. (19), (23), (24), (26), (27), and (29). We conclude that Eqs. (24) and (26) can be used in the range $0<y<\frac{2}{3} \Phi_{0}$ and Eq. (27) can be used for $k T<y<\frac{2}{3} \Phi_{0}$. Note that according to Eq. (27), after ceasing illumination the SPV decrease becomes noticeable after a duration of time $\tau$, which strongly depends on the band bending established during illumination, i.e., on $\Phi=\Phi_{0}-y_{0}$ [compare Figs. 2(b) and 2(c)].

\section{EXPERIMENTAL}

A number of undoped $\mathrm{GaN}$ layers with thicknesses in the range of $1-3 \mu \mathrm{m}$ were grown on $c$-plane sapphire by $\mathrm{MBE}$ and MOCVD. The electron carrier concentration at room temperature, determined from Hall effect measurements, varied from $10^{16}$ to $10^{19} \mathrm{~cm}^{-3}$. In selected samples the concentration of uncompensated shallow donors $N_{D}$ was estimated 

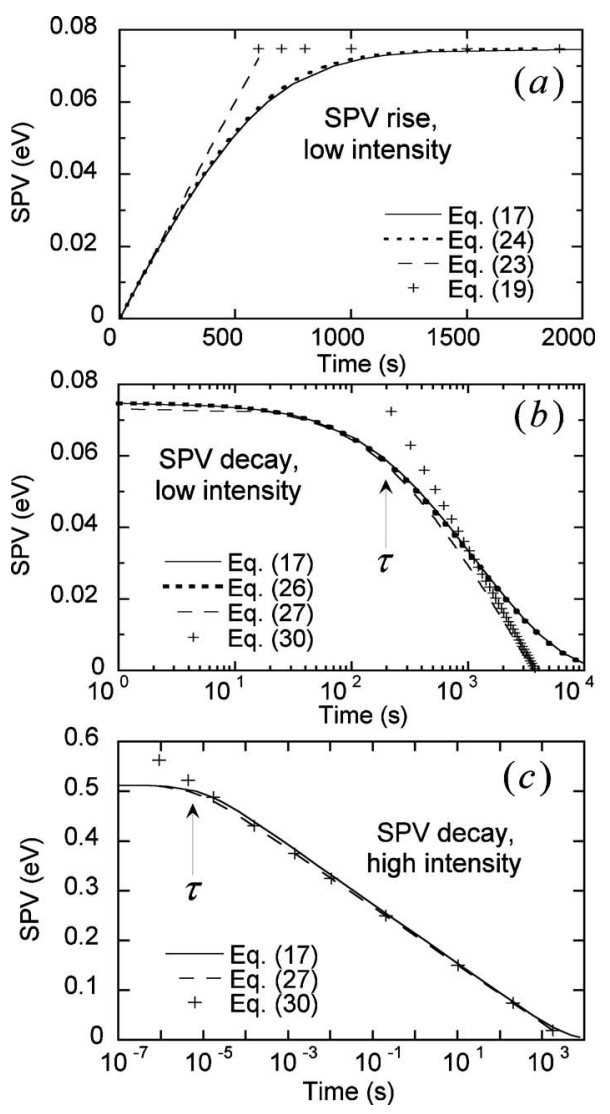

FIG. 2. SPV transients calculated with approximate equations and from numerical solution of Eq. (17). (a) SPV rise [the steady-state SPV calculated using Eq. (19) is also included], $P_{0}=1.8 \times 10^{9} \mathrm{~cm}^{-2} \mathrm{~s}^{-1}$; (b) SPV decay after low intensity illumination $\left(P_{0}=1.8 \times 10^{9} \mathrm{~cm}^{-2} \mathrm{~s}^{-1}\right)$; (c) SPV decay after high intensity illumination $\left(P_{0}=6 \times 10^{16} \mathrm{~cm}^{-2} \mathrm{~s}^{-1}\right)$. Other parameters used in the calculations are: $\Phi_{0}=0.9 \mathrm{eV}, \eta=1, k T=0.0254 \mathrm{eV}, R_{0}=2.6$ $\times 10^{6} \mathrm{~cm}^{-2} \mathrm{~s}^{-1}, n_{s}(0)=7 \times 10^{11} \mathrm{~cm}^{-2}, c=0.026$. In Fig. 2(c) the curve calculated with Eq. (26) lies between the curves calculated with Eqs. (17) and (27) and is not shown for clarity. Arrows indicate the value of $\tau$ calculated from Eq. (28) with the above parameters.

from capacitance-voltage measurements. A representative MBE-grown sample with $N_{D}=4 \times 10^{16} \mathrm{~cm}^{-3} \quad(D$ $=2.5 \mu \mathrm{m}, W=0.15 \mu \mathrm{m})$ was chosen for detailed study. The majority of other samples demonstrated similar behavior to this sample. The surface morphology was studied by atomic force microscopy and the contact potential difference $V_{c p}$ was measured using a Kelvin probe.

The Kelvin probe set-up consisted of a UHV Kelvin probe (KP-6500 from McAllister Technical Services) attached to an optical cryostat (VPF-700 from Janis Research Co., Inc.). ${ }^{49}$ This set-up integrates the advantages of high vacuum or a controlled environment, controlled sample temperature in the range from -150 to $350{ }^{\circ} \mathrm{C}$, and access to continuous and pulse monochromatic light with different wavelengths. The oscillating tip of the Kelvin probe $(4 \mathrm{~mm}$ diameter) was brought close to the GaN sample surface which was mounted in a holder with illumination access from the back or front side. To provide a common ground for the sample and probe, an indium Ohmic contact was soldered on the periphery of the sample surface. Prior to illumination, the sample was maintained in dark for an extended period in order to minimize any residual SPV from previous light exposure.

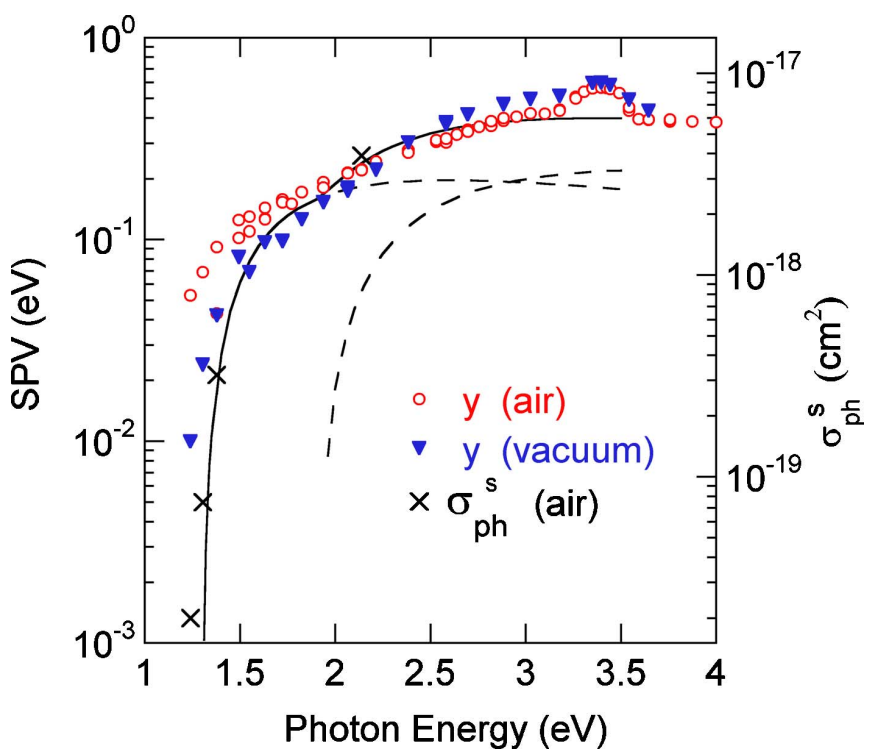

FIG. 3. (Color online) Steady-state photovoltage spectrum from GaN measured at room temperature in vacuum and air, using a light power density of $6 \times 10^{16} \mathrm{~cm}^{-2} \mathrm{~s}^{-1}$. The optical cross-section $\sigma_{p h}^{s}$ is calculated from the $(d y / d t) / P_{0}$ dependences at different wavelengths by using Eq. (25). The two dashed curves are calculated using the Lucovsky model (Ref. 54) for photoionization from two discrete states located 1.3 and $1.8 \mathrm{eV}$ below the conduction band, and the solid curve is their sum.

In most experiments the sample was illuminated from the backside through a sapphire window and the sample sapphire substrate. Light from a $75 \mathrm{~W}$ xenon lamp was filtered through a $0.25 \mathrm{~m}$ grating monochromator and long pass filters. Calibrated neutral-density filters were used to attenuate the standard light power density of about $0.03 \mathrm{~W} / \mathrm{cm}^{2}$ by up to nine orders of magnitude. In the SPV spectra measurements a constant photon flux was achieved by varying the slit width. Wavelengths were changed from 1200 to $300 \mathrm{~nm}$ in varying steps. At each step, the steady-state or an almost saturated condition was achieved. In a few experiments the sample was illuminated with an unfocused $\mathrm{HeCd}$ laser (325 $\mathrm{nm}, 40 \mathrm{~mW}$ ) beam directed to the front sample surface through a special window port in the optical cryostat. In these experiments a semitransparent perforated probe was used. After establishing quantitative agreement in photovoltage values obtained for front and back illumination configurations, we chose the backside geometry for our experiments because of the availability of light with different photon energies and an improved signal-to-noise ratio. SPV measurements were also performed on a semi-transparent, metallized neutral density filter under a variety of conditions to confirm that the effect of photoinduced adsorption or desorption on the steel probe itself was insignificant $(<0.01 \mathrm{eV})$.

\section{RESULTS}

\section{A. SPV spectrum}

Steady-state SPV spectra for the studied GaN sample are shown in Fig. 3 at room temperature in vacuum and air ambient. The SPV was obtained using the backside illumination geometry and had a threshold photon energy of $\sim 1.3 \mathrm{eV}$. After an initial sharp increase, the SPV signal partially saturated between 1.5 and $2 \mathrm{eV}$, had a less distinct step above 2 


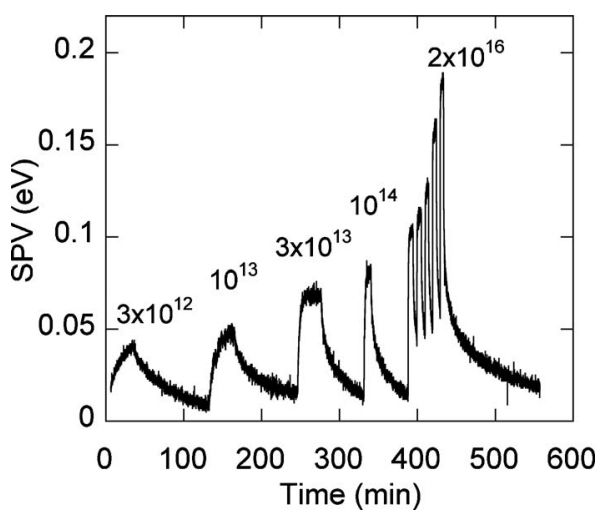

FIG. 4. Room-temperature SPV transients (rise during illumination and decay in dark) obtained in air ambient with below-bandgap illumination (580 $\mathrm{nm}$ ) and at different light intensities (in per square centimeter per second).

$\mathrm{eV}$, and then reached a relatively sharp maximum at $3.4 \mathrm{eV}$, which is close to the $\mathrm{GaN}$ bandgap value at room temperature $(3.43 \mathrm{eV})$. At higher photon energies, the SPV dropped due to a significant rise in the absorption coefficient and a subsequent inability of photons to reach the depletion region in the backside geometry. The SPV signal above $3.5 \mathrm{eV}$ is caused by near-band edge photoluminescence penetrating the sample and being partially absorbed in the depletion region. It can be estimated from the SPV dependence on light intensity (Sec. IV C) that the observed SPV signal at photon energies above the bandgap can be caused by near-band edge photoluminescence with a quantum efficiency of just $0.03 \%$. In vacuum, the long-wavelength edge of the SPV spectrum was steeper than in air, and the second step above $2 \mathrm{eV}$ was slightly larger.

\section{B. Rise of the SPV}

Typical SPV transients for below-bandgap and band-toband illumination are shown in Figs. 4 and 5, respectively. As expected, the SPV rise with time was slow at very low illumination intensities. At higher intensities the SPV increased more steeply and required less time to achieve saturation. The SPV decay after ceasing illumination was slow at any light intensity. To ensure that no accumulative effects were present during these experiments, the SPV was mea-

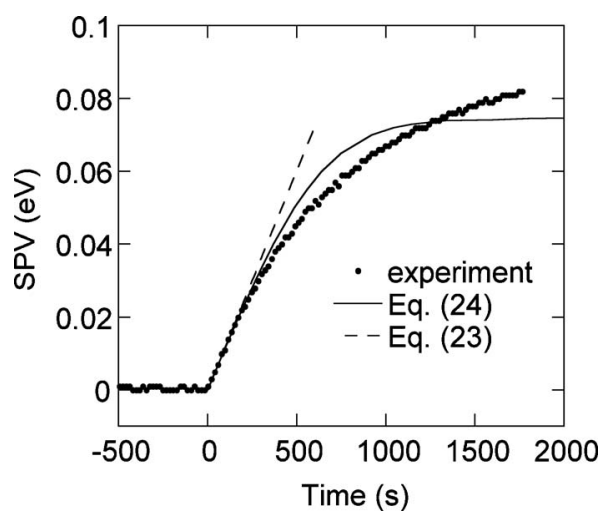

FIG. 5. Photovoltage rise in air ambient under band-to-band illumination $(365 \mathrm{~nm})$. Curves are calculated with Eqs. (23) and (24), using the following parameters: $\Phi_{0}=0.9 \mathrm{eV}, \quad \eta=1, k T=0.0254 \mathrm{eV}, R_{0}=2.6 \times 10^{6} \mathrm{~cm}^{-2} \mathrm{~s}^{-1}$, $n_{s}(0)=7 \times 10^{11} \mathrm{~cm}^{-2}, c=0.026$, and $P_{0}=1.8 \times 10^{9} \mathrm{~cm}^{-2} \mathrm{~s}^{-1}$.

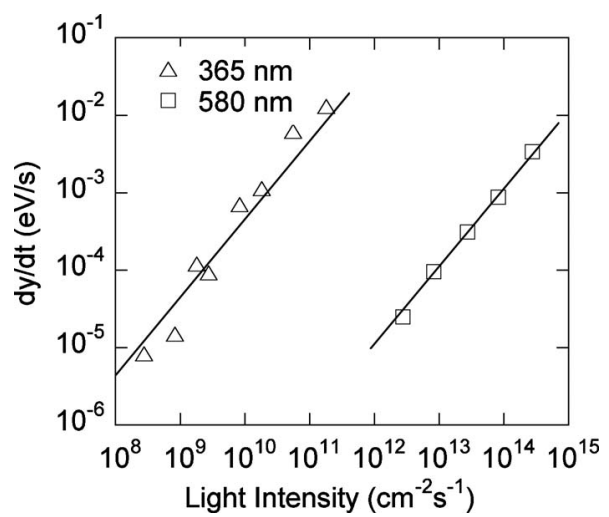

FIG. 6. Initial slope of the $y(t)$ dependence as a function of light power intensity upon switching on illumination at $295 \mathrm{~K}$ in air ambient. Lines show linear dependences.

sured at select light intensities and photon energies after dark conditions were both achieved and not achieved. The signal for the same illumination intensity was reproducible.

The initial slope of the SPV signal, $d y / d t$, is a linear function of the illumination intensity, as shown in Fig. 6. For the dependence at $365 \mathrm{~nm}$, we obtain $(d y / d t) / P_{0}=(5 \pm 2)$ $\times 10^{-14} \mathrm{eV} \mathrm{cm}^{2}$ and $\Phi_{0}=0.8 \pm 0.2 \mathrm{eV}$ using Eq. (23) with $N_{D}=4 \times 10^{16} \mathrm{~cm}^{-3}$ and $c=0.02$ [parameter $c$ was estimated from Eq. (15) for the sample with $D=2.5 \mu \mathrm{m}$ and $W$ $=0.15 \mu \mathrm{m}]$. This $\Phi_{0}$ value is consistent with the largest photovoltage (about $0.6 \mathrm{eV}$ ) for this sample in air ambient and is in agreement with previous reports. ${ }^{4}$ However, since the error may be significant for the estimated number of photons absorbed in the depletion region, i.e., for the value of parameter $c$ in Eq. (23), it is more appropriate to independently estimate the band bending and then determine the value of $c$. Based on the arguments to be given in Sec. IV C, we will slightly correct the above-found value of $\Phi_{0}$ and hereafter will use the following values for the studied $\mathrm{GaN}$ sample: $\Phi_{0}=0.9 \pm 0.2 \mathrm{eV}, c=0.02$, and $n_{s}(0)=7 \times 10^{11} \mathrm{~cm}^{-2}$ [from Eq. (2)]. The optical cross-section for the surface states is then calculated as $\sigma_{p h}^{s}(580 \mathrm{~nm})=(4 \pm 1) \times 10^{-18} \mathrm{~cm}^{2}$ using Eq. (25) and the initial slope $d y / d t$ at $580 \mathrm{~nm}(8$ $\times 10^{-18} \mathrm{eV} \mathrm{cm}^{2}$ ). The optical cross-section determined using this method for several photon energies is shown on Fig. 3.

\section{Steady-state SPV}

Figure 7 shows the steady-state SPV dependence on light intensity for select photon energies. At very low illumination intensity, an extended illumination time is required to reach nearly complete saturation of the SPV. Data for intensities between $10^{8}$ and $10^{10} \mathrm{~cm}^{-2} \mathrm{~s}^{-1}$ required durations up to $1 \mathrm{~h}$ using $365 \mathrm{~nm}$ illumination. With increasing intensity, the time required for nearly complete saturation decreased and the initial $d y / d t$ values correspondingly increased (Fig. 6). In Fig. 7, the $y_{0}\left(P_{0}\right)$ dependences were fitted using Eq. (19) (solid curves) and could be fitted in a wide region of $P_{0}$ with a simple logarithmic dependence (dotted curves). For band-to-band light $(365 \mathrm{~nm})$, an apparent saturation of the SPV signal begins at $P_{0}>10^{-3} \mathrm{~W} / \mathrm{cm}^{2}\left(2 \times 10^{15} \mathrm{~cm}^{-2} \mathrm{~s}^{-1}\right)$ and a maximum value of $y_{0}=0.6 \mathrm{eV}$ occurs at $P_{0}$ $=0.03 \mathrm{~W} / \mathrm{cm}^{2}\left(6 \times 10^{16} \mathrm{~cm}^{-2} \mathrm{~s}^{-1}\right)$. The experimental points 


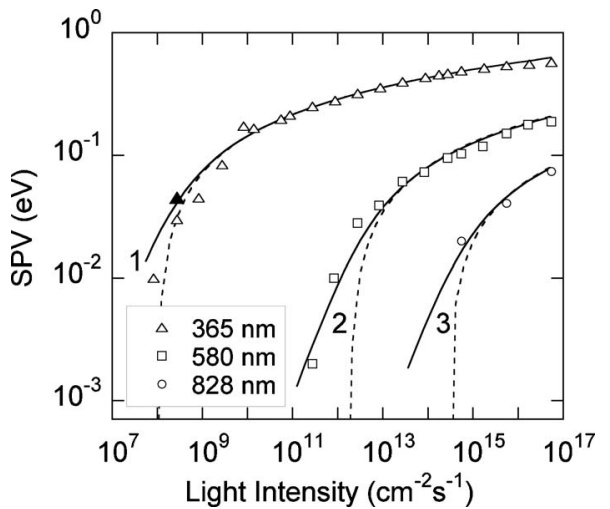

FIG. 7. Dependence of the photovoltage on light intensity in air ambient. Solid curves are fit with Eq. (19) using fitting parameters $\eta$ and $c / R_{0}$ as follows: $\eta=1.23$ (curve 1), 0.8 (curve 2), and 0.6 (curve 3); $c / R_{0}\left(\mathrm{~cm}^{2} \mathrm{~s}\right.$ ) $=1 \times 10^{-8}$ (curve 1 ), $5 \times 10^{-13}$ (curve 2), and $4 \times 10^{-15}$ (curve 3 ). Dotted curves are calculated using the same parameters but with omission of the 1 in Eq. (19). Note, that the solid triangle $(365 \mathrm{~nm})$ at low light intensity was given larger weight since it was obtained after $3 \mathrm{~h}$ of exposure when complete saturation was reached (as compared to $1 \mathrm{~h}$ exposure for points in the vicinity).

were fitted to Eq. (19) with the parameters given in the caption to Fig. 7. An important parameter of the model is the transfer rate $R_{0}$ of electrons from the bulk to surface under dark conditions. We find that $R_{0}=2 \times 10^{6} \mathrm{~cm}^{-2} \mathrm{~s}^{-1}$ using $c$ $=0.02$ for $365 \mathrm{~nm}$ light. The fitting of the $y_{0}\left(P_{0}\right)$ data using Eq. (19) at different wavelengths with this $R_{0}$ value allows us to determine the parameter $c$ as a function of photon energy. Using this information and Eq. (16), the spectral dependence of the optical cross-section $\sigma_{p h}^{s}(\hbar \omega)$ can then be found. This method yields the optical cross-section for the surface states of $1.1 \times 10^{-20} \mathrm{~cm}^{2}$ at $1.5 \mathrm{eV}(828 \mathrm{~nm})$ and $1.4 \times 10^{-18} \mathrm{~cm}^{2}$ at $2.1 \mathrm{eV}(580 \mathrm{~nm})$. These values are lower than those found from the initial rate of the $y(t)$ dependence (Fig. 3), particularly at $828 \mathrm{~nm}$. Note, however, that in different runs the $y_{0}$ at $828 \mathrm{~nm}$ varied between 80 and $125 \mathrm{meV}$ at highest illumination level, which corresponds to a variation in the calculated $\sigma_{p h}^{s}(828 \mathrm{~nm})$ from $1 \times 10^{-20}$ to $2 \times 10^{-19} \mathrm{~cm}^{2}$. Consequently, the accuracy of $\sigma_{p h}^{s}$ determined from the logarithmic $y_{0}\left(P_{0}\right)$ dependence is low, especially close to the threshold photon energies where the SPV signal is weak. Therefore, the $\sigma_{p h}^{s}(\hbar \omega)$ values obtained from the initial values should be considered as more reliable data.

Assuming that $\Phi_{0}=0.9 \mathrm{eV}$ and $R_{0}=2 \times 10^{6} \mathrm{~cm}^{-2} \mathrm{~s}^{-1}$, we can estimate the effective electron capture characteristics of the surface states as: $C_{n 1} \approx 5 \times 10^{-9} \mathrm{~cm}^{3} / \mathrm{s}, \quad s_{n} \approx 5$ $\times 10^{5} \mathrm{~cm} / \mathrm{s}, \quad C_{n 2} \approx 1.3 \times 10^{-9} \mathrm{~cm}^{3} / \mathrm{s}, \quad \sigma_{n 1} \approx 6 \times 10^{-17} \mathrm{~cm}^{2}$, and $\sigma_{n 2} \approx 8 \times 10^{-15} \mathrm{~cm}^{2}$. These values are obtained from Eqs. (9), (11), and (12) using $N_{C}=2.5 \times 10^{18} \mathrm{~cm}^{-3}, v_{n}=2.3$ $\times 10^{7} \mathrm{~cm} / \mathrm{s}, N_{s}^{*}=10^{14} \mathrm{~cm}^{-2}, E_{C}-F=0.1 \mathrm{eV}$, and $n_{s}(0)=7$ $\times 10^{11} \mathrm{~cm}^{-2}$. Note, that the values of $C_{n}, \sigma_{n}$, and $s_{n}$ are much more sensitive to the value of $\Phi_{0}$ than to uncertainties in other variables. For instance, $C_{n}, \sigma_{n}$, and $s_{n}$ change by a factor of $\approx 10^{7}$ within the range of $\Phi_{0}=0.9 \pm 0.2 \mathrm{eV}$. Therefore, the above calculations do not allow us to precisely determine the values of $C_{n}, \sigma_{n}$, or $s_{n}$. On the other hand, assuming that $\sigma_{n} \approx 10^{-16} \mathrm{~cm}^{2}$, which is on the order of atomic dimensions, we obtain $\Phi_{0} \approx 0.9 \mathrm{eV}$ for the GaN sample under study.

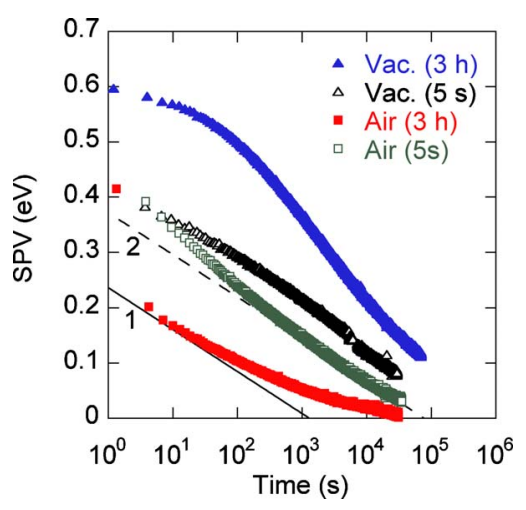

FIG. 8. (Color online) Decay of the photovoltage after band-to-band illumination $(365 \mathrm{~nm})$ at $P_{0}=6 \times 10^{16} \mathrm{~cm}^{-2} \mathrm{~s}^{-1}\left(0.03 \mathrm{~W} / \mathrm{cm}^{2}\right)$ for $5 \mathrm{~s}$ and $3 \mathrm{~h}$ in both vacuum and air ambient. $y_{0}=0.64 \mathrm{eV}$ and $0.46 \mathrm{eV}$ in air for $5 \mathrm{~s}$ and 3 $\mathrm{h}$, respectively, and $y_{0}=0.56 \mathrm{eV}$ and $0.63 \mathrm{eV}$ in vacuum for $5 \mathrm{~s}$ and $3 \mathrm{~h}$, respectively. Curves 1 and 2 are calculated with Eq. (27) using the following parameters: $y_{0}=0.6 \mathrm{eV}, \eta=1.23$, and $\tau=38 \mu \mathrm{s}$ (curve 1 , corresponds to $R_{0}=2 \times 10^{6} \mathrm{~cm}^{-2} \mathrm{~s}^{-1}$ ) and $2 \mathrm{~ms}$ (curve 2, corresponds to $R_{0}=4$ $\left.\times 10^{4} \mathrm{~cm}^{-2} \mathrm{~s}^{-1}\right)$.

The evolution of the SPV signal in different ambients and at highest illumination intensity was reported in Ref. 50. We observed an interesting effect upon illumination with band-to-band light at room temperature. In air ambient and at the highest light intensity, the SPV reached a maximum of $0.62 \mathrm{eV}$ in the first few seconds and then decreased by almost $0.3 \mathrm{eV}$ under UV exposure for $3 \mathrm{~h}$. This quenching effect decreased with decreasing light intensity and could not be observed for $P_{0}<10^{-3} \mathrm{~W} / \mathrm{cm}^{2}$. This dependence can be fitted with Eq. (27) using $\eta=1.9$ and $\tau=50 \mathrm{~s}$, although the meaning of the constants may be different from their use in equations describing SPV decay in dark [Eqs. (27)-(30)]. In contrast to ambient behavior, the SPV in vacuum initially jumped to $0.53 \mathrm{eV}$ and then gradually increased in time and saturated at about $0.64 \mathrm{eV}$ after $3 \mathrm{~h}$ of UV exposure. As described elsewhere, these environmental effects can be explained qualitatively by the photoinduced adsorption of oxygen species in air ambient and the desorption of such species in vacuum under UV illumination. ${ }^{50}$

\section{Decay of the SPV}

The SPV decay transients in the case of band-to-band and below-bandgap illumination after switching off the highest intensity illumination $\left(P_{0}=6 \times 10^{16} \mathrm{~cm}^{-2} \mathrm{~s}^{-1}\right)$ are shown in Figs. 8 and 9, respectively. After illumination with $365 \mathrm{~nm}$ light for a few seconds, the SPV decayed nearly logarithmically with similar rates in vacuum and air ambient (Fig. 8). However, after prolonged exposure, the SPV signals in vacuum and air decayed in substantially different manners. In vacuum, the decay is very slow and the dark signal cannot be reached even after several days in dark. In air, the SPV signal drops from 0.4 to $0.2 \mathrm{eV}$ in about $5 \mathrm{~s}$ and then decays nearly logarithmically to reach its dark value in about $1 \mathrm{~h}$. Using the model developed in Sec. II, we can examine the decays after short $(5 \mathrm{~s})$ illumination times for which contributions from slow processes such as photoadsorption and photodesorption $^{50}$ are minimized. By using the $R_{0}$ value (2 $\left.\times 10^{6} \mathrm{~cm}^{-2} \mathrm{~s}^{-1}\right)$ found from the fit of the $y_{0}\left(P_{0}\right)$ dependence 


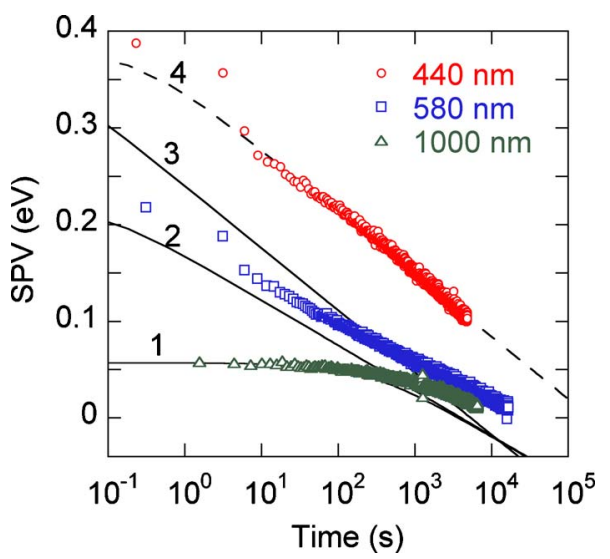

FIG. 9. (Color online) Decay of the photovoltage after below-bandgap illumination at $P_{0}=6 \times 10^{16} \mathrm{~cm}^{-2} \mathrm{~s}^{-1}$ in air ambient. Curves 1 to 4 are fit with Eq. (27) using the following parameters: $y_{0}=0.057 \mathrm{eV}$ (curve 1), $0.22 \mathrm{eV}$ (curve 2), $0.38 \mathrm{eV}$ (curves 3 and 4); $\eta=0.8$ (curves 1 and 2) and 1.1 (curves 3 and 4); $\tau=240 \mathrm{~s}$ (curve 1), $0.08 \mathrm{~s}$ (curve 2), $0.007 \mathrm{~s}$ (curve 3), and $0.25 \mathrm{~s}$ (curve 4). Curves 1 to 3 correspond to $R_{0}=2 \times 10^{6} \mathrm{~cm}^{-2} \mathrm{~s}^{-1}$, and curve 4 to $R_{0}=5 \times 10^{4} \mathrm{~cm}^{-2} \mathrm{~s}^{-1}$.

with Eq. (19) and other above-estimated parameters $\left(y_{0}\right.$ $\left.=0.6 \mathrm{eV}, \Phi_{0}=0.9 \mathrm{eV}, n_{s}(0)=7 \times 10^{11} \mathrm{~cm}^{-2}\right)$, we find from Eq. (28) that the characteristic time of logarithmic decay is expected to be $\tau \approx 40 \mu \mathrm{s}$. Surprisingly, Eq. (27) with the predicted parameter $\tau$ corresponds to a much faster SPV decay (curve 1 in Fig. 8) than that observed experimentally. In order to fit the SPV decay after short exposure with $365 \mathrm{~nm}$ light, parameter $\tau$ should be about $2 \mathrm{~ms}$ (corresponds to $R_{0}$ $\approx 4 \times 10^{4} \mathrm{~cm}^{-2} \mathrm{~s}^{-1}$ ), see curve 2 in Fig. 8 . This discrepancy between the expected and experimentally observed SPV decay is caused by the contribution of external mechanisms to the SPV at high illumination intensity, as will be discussed in Sec. V D.

Figure 9 shows the SPV decay behavior after illumination with below-bandgap light, which results in the resonant excitation of surface states. The SPV decay in dark was also fitted using Eq. (27) with $y_{0}$ equal to the steady-state SPV signal before switching off the light and with the parameters estimated above $\left(R_{0}=2 \times 10^{6} \mathrm{~cm}^{-2} \mathrm{~s}^{-1}, \Phi_{0}=0.9 \mathrm{eV}, n_{s}(0)\right.$ $=7 \times 10^{11} \mathrm{~cm}^{-2}$ ). The results are shown in Fig. 9 as the solid curves. The agreement between the calculated curves and experimental data is reasonable at $1000 \mathrm{~nm}$ (curve 1) and $580 \mathrm{~nm}$ (curve 2) but is poor at $440 \mathrm{~nm}$ (curve 3) and can be improved only when the $\tau$ parameter is increased by a factor of 40 (curve 4). Therefore, the discrepancy in parameter $\tau$ (or $R_{0}$ ) estimated from the $y_{0}\left(P_{0}\right)$ dependence and from fitting to the SPV decay increases when approaching the band gap, which is consistent with the behavior at $365 \mathrm{~nm}$ (Fig. 8).

In order to establish whether the above-mentioned discrepancy is related to photon energy and/or illumination intensity, we examined the SPV decay after switching off the light at 365 and $580 \mathrm{~nm}$ in a wide range of illumination intensities. The agreement between the calculated and measured dependences improves with decreasing illumination level for both band-to-band $(365 \mathrm{~nm})$ and below-bandgap $(580 \mathrm{~nm})$ light. On the other hand, an excellent agreement between experimental data and calculated curves is observed when $R_{0}$ is treated as a fitting parameter that may depend on

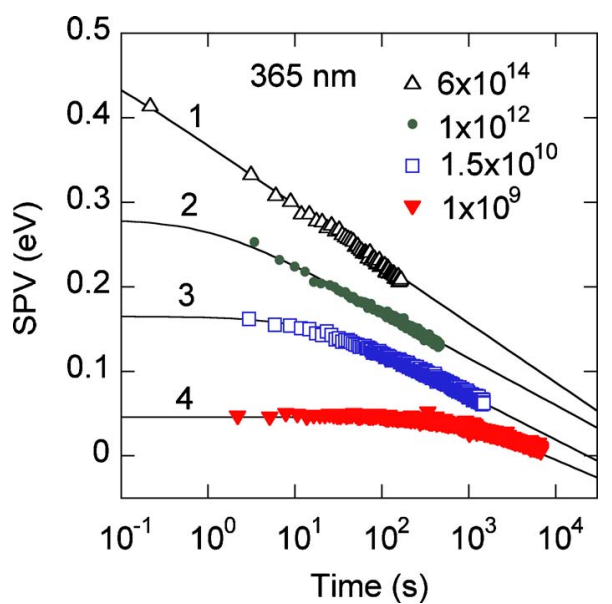

FIG. 10. (Color online) Decay of the photovoltage after band-to-band illumination $(365 \mathrm{~nm})$ in air ambient at room temperature for different light power densities $P_{0}\left(\mathrm{~cm}^{-2} \mathrm{~s}^{-1}\right)=6 \times 10^{14}$ (empty triangles), $1 \times 10^{12}$ (solid circles), $1.5 \times 10^{10}$ (empty squares), and $1 \times 10^{9}$ (solid triangles). The solid curves are fit with Eq. (27) using $R_{0}=6.5 \times 10^{4} \mathrm{~cm}^{-2} \mathrm{~s}^{-1}$ (curve 1), 8.1 $\times 10^{4} \mathrm{~cm}^{-2} \mathrm{~s}^{-1}$ (curve 2), $3.8 \times 10^{5} \mathrm{~cm}^{-2} \mathrm{~s}^{-1}$ (curve 3), $1 \times 10^{6} \mathrm{~cm}^{-2} \mathrm{~s}^{-1}$ (curve 4). Other parameters include: $y_{0}=0.49 \mathrm{eV}$ (curve 1), $0.28 \mathrm{eV}$ (curve 2), $0.165 \mathrm{eV}$ (curve 3), and $0.046 \mathrm{eV}$ (curve 4); $\eta=1.2$ (curve 1), 0.95 (curve 2), 0.9 (curve 3), and 0.8 (curve 4). $\Phi_{0}=0.9 \mathrm{eV}$ and $n_{s}(0)=7 \times 10^{11} \mathrm{~cm}^{-2}$ for all the curves.

illumination intensity (Fig. 10). The $R_{0}$ parameters obtained from fits of decay curves (365 and $580 \mathrm{~nm}$ ) to Eq. (27) are shown in Fig. 11. The figure also includes the value of $R_{0}$ determined from fitting the $y_{0}\left(P_{0}\right)$ dependence with Eq. (19) (dashed curve) and the value of $R_{0}$ determined from fitting the SPV rise (solid triangle) with Eq. (24) in Fig. 5. For band-to-band light at low illumination intensity $\left(<10^{11} \mathrm{~cm}^{-2} \mathrm{~s}^{-1}\right)$, the $R_{0}$ determined from SPV decay curves is close to the $R_{0}$ determined from fitting $y_{0}\left(P_{0}\right)$ and $y(t)$ dependences with Eqs. (19) and (24), respectively. How-

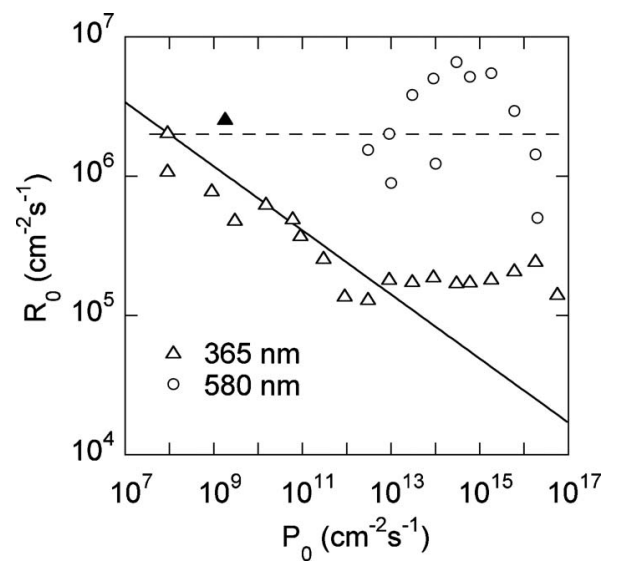

FIG. 11. Parameter $R_{0}$ determined from the photovoltage decays after illumination with $365 \mathrm{~nm}$ (empty triangles) and $580 \mathrm{~nm}$ light (empty circles) with different intensities. $R_{0}$ is found from the fit of decay curves with Eq. (27) and by taking $\Phi_{0}=0.9 \mathrm{eV}$ and $n_{s}(0)=7 \times 10^{11} \mathrm{~cm}^{-2} \cdot \eta$ is the fitting parameter which gradually increases from 0.8 to 1.25 for the $365 \mathrm{~nm}$ light intensity increasing from $10^{8}$ to $6 \times 10^{16} \mathrm{~cm}^{-2} \mathrm{~s}^{-1}$ and equals to 0.8 for the $580 \mathrm{~nm}$ light. The dashed horizontal line indicates the value of $R_{0}$ found from the $y_{0}\left(P_{0}\right)$ dependence for $365 \mathrm{~nm}$ illumination. The solid triangle shows $R_{0}$ found from the rise of the SPV for $365 \mathrm{~nm}$ illumination (Fig. 5). The solid line is $R_{0}^{e f f}$ calculated by using Eq. (36) with $R_{0}=2$ $\times 10^{6} \mathrm{~cm}^{-2} \mathrm{~s}^{-1}$ and $\eta=1.23$. 


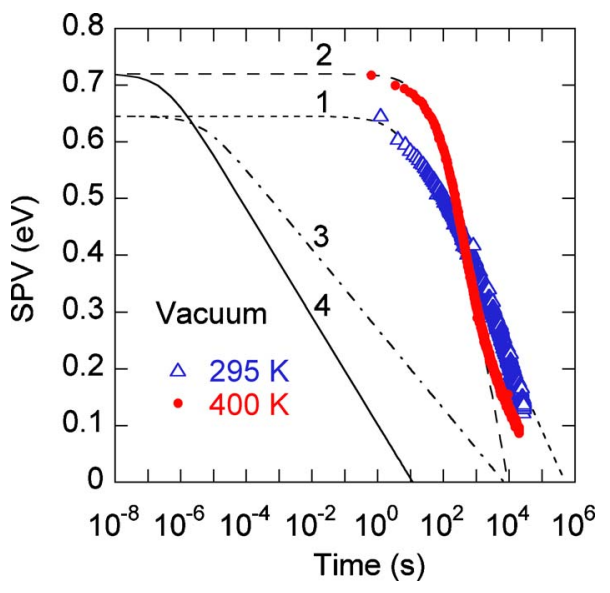

FIG. 12. (Color online) Decay of the photovoltage after band-to-band illumination $(365 \mathrm{~nm})$ in vacuum at $295 \mathrm{~K}$ (triangles) and $400 \mathrm{~K}$ (circles) with $P_{0}=0.03 \mathrm{~W} / \mathrm{cm}^{2}$ for more than one hour. The curves are fit with Eq. (27) using the following parameters: $T=295 \mathrm{~K}$ (curves 1 and 3) and $400 \mathrm{~K}$ (curves 2 and 4); $y_{0}=0.64 \mathrm{eV}$ (curves 1 and 3 ) and $0.72 \mathrm{eV}$ (curves 2 and 4); $\Phi_{0}=0.9 \mathrm{eV}$ (curves 1 and 3) and $1.0 \mathrm{eV}$ (curves 2 and 4); $E_{C}-F$ $=0.1 \mathrm{eV}$ (curves 1 and 3) and $0.15 \mathrm{eV}$ (curves 2 and 4); $\eta=2.2$ (curve 1), 4.0 (curve 2), and 1.2 (curves 3 and 4); $\tau=5 \mathrm{~s}$ (curve 1), $50 \mathrm{~s}$ (curve 2), $4.5 \times 10^{-6} \mathrm{~s}$ (curve 3), and $3.1 \times 10^{-7} \mathrm{~s}$ (curve 4). Parameters for curves 1 and 2 correspond to $R_{0}=4.6 \times 10^{4} \mathrm{~cm}^{-2} \mathrm{~s}^{-1}$ and $5.4 \times 10^{6} \mathrm{~cm}^{-2} \mathrm{~s}^{-1}$, respectively. Parameters for curves 3 and 4 correspond to $R_{0}=2 \times 10^{6} \mathrm{~cm}^{-2} \mathrm{~s}^{-1}$ and $1.2 \times 10^{9} \mathrm{~cm}^{-2} \mathrm{~s}^{-1}$, respectively.

ever, at high illumination intensity the discrepancy in $R_{0}$ determined from independent methods increases.

\section{E. Effect of temperature}

The absolute value of the surface contact potential difference decreased by $0.15 \mathrm{~V}$ as the sample temperature was increased from 295 to $400 \mathrm{~K}$ in vacuum, indicating that the upward band bending at the surface increased by this value. The maximum SPV for $365 \mathrm{~nm}$ at $P_{0}=0.03 \mathrm{~W} / \mathrm{cm}^{2}$ increased from 0.64 to $0.72 \mathrm{eV}$. We compared the decay of the SPV signal after switching off the $365 \mathrm{~nm}$ light at 295 and $400 \mathrm{~K}$ (Fig. 12). The SPV decay in vacuum at 295 and $400 \mathrm{~K}$ is much slower than predicted from Eq. (27) with parameters $R_{0}$ or $\tau$ predicted from the analysis of the $y_{0}\left(P_{0}\right)$ dependence (curves 3 and 4 in Fig. 12). At $400 \mathrm{~K}$, the SPV decay starts at $\tau \approx 50 \mathrm{~s}$, and the slope of the logarithmic decay is much larger than expected. Although the dependence can be formally fitted with Eq. (27) (curve 2 in Fig. 12), it reveals unreasonably large value of $\eta$. Such large $\eta$ values and the large $\tau$ values at 295 and $400 \mathrm{~K}$ may indicate that surface charge restoration after ceasing illumination in vacuum is a more complex process than used in the model presented in Sec. II. The slow photodesorption of oxygen species in vacuum during UV illumination and the slow adsorption of these species after ceasing illumination cannot be ignored in this case. ${ }^{50}$

\section{DISCUSSION}

\section{A. Determination of band bending in dark}

It is difficult to reliably determine the absolute value of band bending $\Phi_{0}$ in dark. When this parameter is determined from photoelectron spectroscopy by measuring the binding (a) dark

(b) air

(c) vacuum

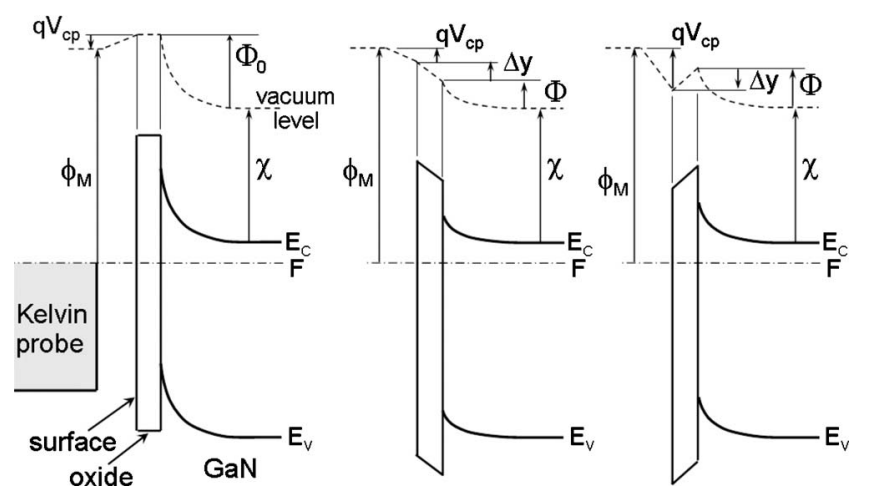

FIG. 13. Schematic band diagram, including a semiconductor, native oxide layer, and a Kelvin probe. (a) Dark conditions; (b) after extended UV illumination in air; and (c) after extended UV illumination in vacuum. The SPV decay after short UV illumination in air or vacuum can also be explained by diagram (c) but with smaller $\Delta y$.

energies of electron states near the surface, the results can be misleading because the source intensity is oftentimes sufficient to create an SPV signal. ${ }^{11}$ An estimation of band bending at the semiconductor surface in dark using Kelvin probe measurements ${ }^{4,13}$ may also be unreliable since it does not take into account the surface microscopic dipole and uses probe and reference metal work functions that may depend on the ambient. In particular, freshly prepared Pt has shown a contact potential difference of $-1.94 \mathrm{~V}$ when measured using a stainless steel probe but a value of $-0.85 \mathrm{~V}$ under ambient conditions. ${ }^{51}$ Nevertheless, we have estimated that $\Phi_{0}$ in the studied GaN sample equals $0.6 \mathrm{eV}$ at $295 \mathrm{~K}$ in air ambient and $0.7 \mathrm{eV}$ at $400 \mathrm{~K}$ in vacuum using the following equation (see Fig. 13) (Ref. 4):

$$
\Phi=\phi_{M}-\chi-\Delta y-\left(E_{C}-F\right)-q V_{c p},
$$

where $\phi_{M}=3.8 \mathrm{eV}$ for the workfunction of the steel Kelvin probe, ${ }^{51} \chi=3.2 \pm 0.3 \mathrm{eV}$ for the electron affinity of $\mathrm{GaN},{ }^{1,3,52} E_{C}-F=0.1 \mathrm{eV}$ at $295 \mathrm{~K}$ and $0.15 \mathrm{eV}$ at $400 \mathrm{~K}$, and $q V_{c p}=-0.1 \mathrm{eV}$ at $295 \mathrm{~K}$ (as measured both in vacuum and air ambient) and $-0.25 \mathrm{eV}$ at $400 \mathrm{~K}$ (in vacuum) for typical dark values. The value $\Delta y$ is the change in potential across the native oxide layer (Fig. 13), which is assumed to be zero in dark but may appear under illumination, as will be discussed later. Independently, the absolute value of $\Phi_{0}$ can be estimated from the SPV saturation value under intense illumination, when complete or nearly complete flattening of the surface band bending is achieved. ${ }^{53}$ While interpretation of the SPV saturation should be done with caution, ${ }^{18}$ at least the lowest limit of $\Phi_{0}$ can be found at highest illumination intensity when the reduction in band bending is the largest. Using the highest observed SPV at $P_{0}=0.03 \mathrm{~W} / \mathrm{cm}^{2}$, we conclude that $\Phi_{0}>0.65 \mathrm{eV}$ at $295 \mathrm{~K}$ and $\Phi_{0}>0.72 \mathrm{eV}$ at $400 \mathrm{~K}$. However, the most reliable value of $\Phi_{0}$ can be obtained from the steady-state $y_{0}\left(P_{0}\right)$ dependence [Eq. (19)] by varying model parameters within a range of reasonable values and obtaining a reliable $R_{0}$ value. Using the experimentally found value of $R_{0}\left(2 \times 10^{6} \mathrm{~cm}^{-2} \mathrm{~s}^{-1}\right)$ and a reasonable range of the surface recombination velocity $s_{n}=C_{n 1} N_{s}^{*}$ $=10^{1}-10^{9} \mathrm{~cm} / \mathrm{s}$ with a mean value of $\approx 10^{5} \mathrm{~cm} / \mathrm{s}$, we can 
estimate from Eq. (9), that $\Phi_{0}=0.9 \pm 0.2 \mathrm{eV}$ at $295 \mathrm{~K}$. This value is in excellent agreement with the lower limit of $\Phi_{0}$ stated above and should be regarded as the most accurate value.

\section{B. Parameters of the surface states}

From the initial rate of the SPV rise under band-to-band illumination we can reliably determine the density of holes generated in the depletion region and accumulated at the surface per second $\left(R^{*}\right)$ and the parameter $c$ related to the experimental geometry. For below-bandgap illumination this rate provides the value of the optical cross-section of surface states $\sigma_{p h}^{s}(\hbar \omega)$ and its spectral dependence. We have fit the photoionization spectrum using a simple Lucovsky model, ${ }^{54}$ neglecting any long-range coulomb potential, contributions from different bands, and thermal broadening. An example of such a fit is illustrated in Fig. 3, where the two dashed curves show the photoionization cross-section spectra for two discrete levels located at 1.3 and $1.8 \mathrm{eV}$ below the conduction band minimum, and the solid curve is their sum. Note that this fit is only an example illustrating that two discrete surface states are consistent with the observed SPV spectrum; however, a similar spectrum could be constructed by the superposition of spectra from several levels or a continuous distribution of surface states. Moreover, a model that accounts for the temperature dependence, electron-phonon coupling strength, and possible repulsive or attractive character of the surface states could change the shape of the photoionization cross-section spectrum. ${ }^{55}$ The optical cross-section for these surface states, estimated from the initial slope of the $d y / d t\left(P_{0}\right)$ dependence for below-bandgap excitation [Eq. (25)], is in the mid $10^{-18} \mathrm{~cm}^{2}$ range. Although possible contributions to the SPV spectrum from other discrete or continuously distributed states cannot be ruled out, the SPV value under continuous illumination and its transients are relatively insensitive to the energy distribution of the surface states. Indeed, the term $R_{s b}$ in Eq. (13) plays a minor role in all of the experiments and can be ignored when $E_{C}-E_{S}$ $>1 \mathrm{eV}$. Moreover, the contribution from different surface states in capturing electrons from the bulk is accounted for in our model by the introduction of an effective capture coefficient $C_{n 1}$ for the states with effective density $N_{s}^{*}$, or the effective surface recombination velocity $s_{n}$. These parameters may be close to the parameters of the dominant surface state or may be considered as weighted average parameters.

The main parameter of our model is $R_{0}$ which equals the bulk-to-surface flow rate of electrons in dark and their subsequent capture. It strongly depends on the electron capture cross-section of the surface states and the height of the nearsurface barrier. Competition between this rate and the carrier generation rate due to illumination governs the SPV value under continuous illumination. Note that the rate $R_{0}$ is independent of the source of the SPV, i.e., above or belowbandgap excitation. As a result, even without knowing exact parameters of the electron transfer, (e.g., electron capture cross-section, barrier height, and surface state density), the effective optical cross-section $\sigma_{p h}^{s}$ of the surface states can be determined from the $y_{0}\left(P_{0}\right)$ dependences for different photon energies using the value of $R_{0}$ found from the $y_{0}\left(P_{0}\right)$ behavior at $365 \mathrm{~nm}$ (see Sec. IV C). Independently and more accurately, $\sigma_{p h}^{s}$ can be estimated from the rate of SPV rise for certain wavelengths of below-bandgap light (see Sec. IV B). We obtained $\sigma_{p h}^{s}=1 \times 10^{-18} \mathrm{~cm}^{2}$ and $4 \times 10^{-18} \mathrm{~cm}^{2}$ at 580 $\mathrm{nm}$ using the first and second methods, respectively.

It is important to note that the electron capture crosssection for the surface states, $C_{n 1}$, cannot be found from the SPV measurements with any reasonable accuracy. This is because a relatively small amount of uncertainty in the value of dark band bending $\Phi_{0}$, which is unavoidable, causes orders of magnitude uncertainty in the electron capture crosssection. On the other hand, by taking the electron capture cross-section or the surface recombination velocity in a range of reasonable values and using the experimentally found value of $R_{0}$, one can estimate from Eq. (9) the dark band bending value with relatively high accuracy.

\section{Effect of temperature}

In our experimental studies, the band bending increased with increasing temperature, as supported by the decrease in dark contact potential difference and increase in steady-state SPV. This observation agrees with the findings of Long and Bermudez ${ }^{11}$ who used photoelectron spectroscopy to determine that band bending increases with temperature for both $n$-type and $p$-type doping. We found that as temperature increased from 295 to $400 \mathrm{~K}$, the barrier height $\Phi_{0}$ increased by $0.1 \mathrm{eV}$ and the SPV signal in vacuum increased from 0.64 to $0.72 \mathrm{eV}$. In our model, if the surface charge density does not change with temperature, then we would expect $\Phi_{0}$ to be independent of temperature [Eq. (1)], or to slightly decrease with increasing temperature as a consequence of the increased $E_{C}-F$ value if the Fermi level were pinned. We also expect the SPV signal to be nearly constant or to slightly decrease with increasing temperature, as can be shown by substitution of Eq. (9) into Eq. (19) with the factor of one ignored in Eq. (19). If the surface charge in fact depends on temperature due to an external SPV mechanism, we would expect that heating promotes the desorption of surface species in vacuum under UV illumination, which would also result in a decrease in both band bending and SPV with increasing temperature. In both cases, $\Phi_{0}$ and $y$ would be expected to decrease with temperature, in apparent contradiction to experimental data. The contradiction can be resolved by assuming that charge redistributes between external and internal surface states in conditions of intense, extended illumination, as will be discussed below.

\section{Internal and external mechanisms of the SPV}

An interesting result of this work is the discrepancy in some cases between the expected rate of SPV decay after ceasing illumination and the experimentally observed rate. The magnitude of the discrepancy strongly depends on ambient, on duration of the illumination (Fig. 8), and on temperature (Fig. 12). In the following discussion we will distinguish three mechanisms affecting the SPV: (1) an internal mechanism, i.e., accumulation of photogenerated holes at the semiconductor surface and their recombination with elec- 
trons from the bulk passing over the barrier; (2) a relatively fast $\left(\sim 1\right.$ s at high $\left.P_{0}\right)$ redistribution of charge between the semiconductor/oxide interface and oxide after short but intense illumination; and (3) slow photoadsorption and photodesorption processes at the oxide surface after prolonged (hours) illumination with intense UV light.

(1) The internal mechanism is dominant at very low illumination intensity, when all three characteristic regions of the SPV (rise, saturation and decay) are described well for the same value of the parameter $R_{0}$. The $R_{0}$ value determined from the $y_{0}\left(P_{0}\right)$ dependence does not depend on the SPV value, illumination intensity, or ambient, provided that the exposure time is sufficiently long to reach SPV saturation but short enough to ignore photoadsorption and photodesorption processes. When the SPV saturates and the illumination intensity is not too low $\left(y_{0}>k T\right)$, Eq. (13) reduces to $R_{b s} \approx R^{*}$. The parameter $R_{0}$ can then be estimated as $R_{0}=R^{*} \exp \left(-y_{0} / k T\right)$, where $R^{*}$ (flow of holes to surface) can be found from the initial rate of the SPV rise [Eq. (23)]. The $R_{0}$ value determined from the steady-state SPV behavior agrees with the $R_{0}$ value determined from the decay behavior in air at relatively low illumination intensity. We, therefore, conclude that at low intensity the steady-state and transient SPV behaviors arise from a dominant internal mechanism, i.e., the accumulation of holes at semiconductor surface states and their recombination with electrons passing over the barrier. However, the internal mechanism fails to explain the unexpectedly slow SPV decay after illumination with high intensity.

(2) In the case of short and intense UV illumination, the characteristic decay time $\tau$ determined from Eq. (27) is approximately the same for air and vacuum but larger than expected in the case of an internal SPV mechanism (Fig. 8). The observed increase in $\tau$ can be simulated by the formal replacement of $\tau$ with $\tau^{*}$ in Eq. (27), where

$\tau^{*}=\tau \exp (\Delta y / k T)$.

The inclusion of $\Delta y \neq 0$ can be explained physically by the appearance of a potential change $\Delta y$ across the native oxide layer with the more negative potential at the oxide/semiconductor interface [as schematically shown in Fig. 13(c)]. Since this effect is not very sensitive to the ambient, it is reasonable to assume that the redistribution of charge is not related to slow photoinduced processes at the oxide surface. It is likely that the positive charge in the oxide is created by holes leaking from the semiconductor surface to the oxide layer where they recombine with electrons trapped at defects in the oxide. The potential change $\Delta y$ increases with increasing illumination intensity and reaches about $0.1 \mathrm{eV}$ in our experiments.

The replacement of $\tau$ with $\tau^{*}$ is equivalent to an effective reduction in the parameter $R_{0}$ in Eq. (29), or replacement of $R_{0}$ with

$R_{0}^{e f f}=R_{0} \exp (-\Delta y / k T)$.

Assuming that $\Delta y$ changes with the light intensity as
$\Delta y=k T(\eta-1) \ln \left(c P_{0} / R_{0}\right)$,

we can explain not only the decrease in the effective parameter $R_{0}$ with light intensity (solid line in Fig. 11) but also the difference of the phenomenological parameter $\eta$ from unity in Eq. (19). Indeed, Eq. (19) is equivalent to

$y_{0}=k T \ln \left(\frac{c P_{0}}{R_{0}^{e f f}}+1\right)$

with

$R_{0}^{e f f}=R_{0}^{\eta}\left(c P_{0}\right)^{1-\eta}=R_{0} \exp (-\Delta y / k T)$,

at least for the range where " 1 " can be neglected.

(3) After extended illumination with intense UV light, significant changes are observed both in steady-state and transient SPV (Figs. 8 and 12). These changes can be explained qualitatively by the appearance of a positive or negative potential change $\Delta y$ across the oxide layer, which is caused by the photoadsorption of oxygen in air [Fig. 13(b)] or its photodesorption in vacuum [Fig. 13(c)]. Note that photoinduced processes at the oxide surface become noticeable only under intense and extended UV illumination. In this case, the density of electrons and holes reaching the semiconductor surface increases by several orders of magnitude, i.e., by a factor of $\exp (0.6 / 0.0254) \approx 2 \times 10^{10}$ as compared to the density of electrons reaching the surface in dark. (In the case of below-bandgap excitation the density of electrons is relatively low). ${ }^{56}$ These abundant electrons and holes increase the number of carriers tunneling through the thin oxide layer and facilitate surface reactions such as chemisorption. After illumination is ceased, both the potential change $\Delta y$ and the band bending $\Phi$ start to slowly restore to zero and $\Phi_{0}$, respectively.

The slope of the SPV decay for longer times is different from $k T$ because two effects take place simultaneously such as: thermal emission of electrons from the bulk to the oxide/ semiconductor interface over the near-surface barrier, and tunneling of electrons through the thin oxide layer. At $400 \mathrm{~K}$, photodesorption is more efficient and $\Delta y$ is larger. The latter explains the apparent increase in the SPV signal with temperature and is consistent with the fact that the SPV decay at $400 \mathrm{~K}$ starts later than at $295 \mathrm{~K}$ (Fig. 12).

In summary, at low illumination intensity (in any ambient and for both above- and below-bandgap excitation) the SPV in GaN is primarily due to an internal mechanism, and its steady-state value and transients can be quantitatively described within a simple phenomenological model. During short illumination with high-intensity UV light, corrections to the SPV of the order of $0.1 \mathrm{eV}$ may be needed to account for charging of an oxide due to holes leaking from the oxide/ semiconductor interface. Finally, illumination with high intensity for an extended period of time requires external mechanisms such as the photoinduced chemisorption of oxygen in air and the photoinduced desorption of oxygen in vacuum. Our model does not incorporate both internal and external mechanisms simultaneously to reproduce the slow 
variation in SPV under illumination or very slow decays after ceasing illumination. We explain qualitatively the peculiarities of the steady-state and transient SPV in conditions of extended and intense illumination by including a potential drop in a surface oxide layer which increases with illumination intensity. The quantitative simulation of SPV transients for conditions where several mechanisms act simultaneously is beyond the scope of this work.

\section{CONCLUSIONS}

We have investigated the SPV in undoped $n$-type GaN with a Kelvin probe in vacuum and air ambient. The observed dependence of the SPV on illumination intensity, as well as its transients after turning on and off illumination with band-to-band and below-bandgap light, are explained within a suggested phenomenological model. This model accounts for surface-to-bulk and bulk-to-surface electron transfer and the accumulation of photogenerated holes at the surface during illumination. The model explains both the steady-state and transient behavior of the SPV at low illumination intensity. It can be extended to the case of prolonged illumination with intense UV light by accounting for external mechanisms, such as charging of the oxide layer and photoadsorption/desorption of oxygen at the surface. The upward band bending in dark is estimated as $\Phi_{0}$ $=0.9 \pm 0.2 \mathrm{eV}$ at $295 \mathrm{~K}$ and $\Phi_{0}=1.0 \pm 0.2 \mathrm{eV}$ at $400 \mathrm{~K}$. Illumination with photons having an energy close to the bandgap of $\mathrm{GaN}$ reduces this band bending by up to $0.64 \mathrm{eV}$ at $295 \mathrm{~K}$ and $0.72 \mathrm{eV}$ at $400 \mathrm{~K}$. This value increases with a logarithmic law for a wide range of light intensities. The very slow decay of the SPV in dark after ceasing band-toband illumination is qualitatively explained by the contributions of both internal and external mechanisms.

\section{ACKNOWLEDGMENTS}

This work was supported by the National Science Foundation (Grant No. DMR-0804679). The authors are grateful to Dr. H. Morkoç from VCU for providing GaN samples.

${ }^{1}$ V. M. Bermudez, J. Appl. Phys. 80, 1190 (1996).

${ }^{2}$ G. Koley and M. G. Spencer, J. Appl. Phys. 90, 337 (2001).

${ }^{3}$ C. I. Wu, A. Kahn, N. Taskar, D. Dorman, and D. Gallagher, J. Appl. Phys. 83, 4249 (1998).

${ }^{4}$ S. Sabuktagin, M. A. Reshchikov, D. K. Johnstone, and H. Morkoç, in MRS Symposia Proceedings No. 798 (Materials Research Society, Pittsburgh, 2004), p. Y5.39.

${ }^{5}$ D. Goguenheim and M. Lannoo, Phys. Rev. B 44, 1724 (1991).

${ }^{6}$ K. A. Bulashevich and S. Y. Karpov, Phys. Status Solidi C 3, 2356 (2006).

${ }^{7}$ W. E. Spicer, P. W. Chye, P. R. Skeath, C. Y. Su, and I. Lindau, J. Vac. Sci. Technol. 16, 1422 (1979).

${ }^{8}$ H. Hasegawa, L. He, H. Ohno, T. Sawada, T. Haga, Y. Abe, and H. Takahashi, J. Vac. Sci. Technol. B 5, 1097 (1987).

${ }^{9}$ R. A. Beach, E. C. Piquette, and T. C. McGill, MRS Internet J. Nitride Semicond. Res. 4S1, G6.26 (1999).

${ }^{10}$ T. Sasaki and T. Matsuoka, J. Appl. Phys. 64, 4531 (1988).

${ }^{11}$ J. P. Long and V. M. Bermudez, Phys. Rev. B 66, 121308 (2002).

${ }^{12}$ I. Shalish, Y. Shapira, L. Burstein, and J. Salzman, J. Appl. Phys. 89, 390 (2001).

${ }^{13}$ M. A. Reshchikov, S. Sabuktagin, D. K. Johnstone, and H. Morkoç, J. Appl. Phys. 96, 2556 (2004).

${ }^{14}$ I. Shalish, L. Kronik, G. Segal, Y. Rosenwaks, Y. Shapira, U. Tisch, and J. Salzman, Phys. Rev. B 59, 9748 (1999).
${ }^{15}$ D. Mao, A. Khan, M. Marsi, and G. Margaritondo, Phys. Rev. B 42, 3228 (1990).

${ }^{16}$ T. U. Kampen, D. Troost, X. Y. Hou, L. Koenders, and W. Mönch, J. Vac. Sci. Technol. B 9, 2095 (1991).

${ }^{17}$ J. P. Long, H. R. Sadeghi, J. C. Rife, and M. N. Kabler, Phys. Rev. Lett. 64, 1158 (1990).

${ }^{18}$ L. Kronik and Y. Shapira, Surf. Sci. Rep. 37, 1 (1999).

${ }^{19}$ A. Many, Y. Goldstein, and N. B. Grover, Semiconductor Surfaces, 2nd ed. (North-Holland, Amsterdam, 1971).

${ }^{20} \mathrm{~W}$. Mönch, Semiconductor Surfaces and Interfaces, 3rd ed. (Springer, Berlin, 2001), p. 67.

${ }^{21}$ J. Lagowski, C. L. Balestra, and H. C. Gatos, Surf. Sci. 29, 203 (1972).

${ }^{22}$ R. B. Darling, Phys. Rev. B 43, 4071 (1991).

${ }^{23}$ J. Lagowski, C. L. Balestra, and H. C. Gatos, Surf. Sci. 29, 213 (1972).

${ }^{24}$ S. M. Sze, Physics of Semiconductor Devices, 2nd ed. (Wiley, New York, 1981).

${ }^{25}$ E. Yablonovitch, B. J. Skromme, R. Bhat, J. P. Harbison, and T. J. Gmitter, Appl. Phys. Lett. 54, 555 (1989).

${ }^{26}$ L. K. Galbraith and T. E. Fischer, Surf. Sci. 30, 185 (1972).

${ }^{27}$ S. C. Choo, L. S. Tan, and K. B. Quek, Solid-State Electron. 35, 269 (1992).

${ }^{28}$ Q. Liu, H. E. Ruda, G. M. Chen, and M. Simard-Normandin, J. Appl. Phys. 79, 7790 (1996).

${ }^{29}$ E. O. Johnson, Phys. Rev. 111, 153 (1958).

${ }^{30}$ S. C. Dahlberg, J. R. Chelikowsky, and W. A. Orr, Phys. Rev. B 15, 3163 (1977).

${ }^{31}$ A. Morawski, M. M. G. Slusarczuk, J. Lagowski, and H. C. Gatos, Surf. Sci. 69, 53 (1977).

${ }^{32}$ A. M. Goodman, J. Appl. Phys. 32, 2550 (1961).

${ }^{33}$ Z. Z. Bandic, P. M. Bridger, E. C. Piquette, and T. C. McGill, Appl. Phys. Lett. 72, 3166 (1998).

${ }^{34}$ P. M. Bridger, Z. Z. Bandic, E. C. Piquette, and T. C. McGill, Appl. Phys. Lett. 73, 3438 (1998).

${ }^{35}$ K. Kumakura, T. Makimoto, N. Kobayashi, T. Hashizume, T. Fukui, and H. Hasegawa, Appl. Phys. Lett. 86, 052105 (2005).

${ }^{36}$ S. E. Park, J. J. Kopanski, Y.-S. Kang, and L. H. Robins, Phys. Status Solidi C 2, 2433 (2005)

${ }^{37}$ W. H. Brattain and J. Bardeen, Bell Syst. Tech. J. 32, 1 (1953).

${ }^{38}$ R. H. Kingston, J. Appl. Phys. 27, 101 (1956).

${ }^{39}$ J. Lagowski, E. S. Sproles, Jr., and H. C. Gatos, J. Appl. Phys. 48, 3566 (1977).

${ }^{40}$ I. Shalish, L. Kronik, G. Segal, Y. Shapira, M. Eizenberg, and J. Salzman, Appl. Phys. Lett. 77, 987 (2000).

${ }^{41}$ V. Chakrapani, C. Pendyala, K. Kash, A. B. Anderson, M. K. Sunkara, and J. C. Angus, J. Am. Chem. Soc. 130, 12944 (2008).

${ }^{42}$ M. Zafar Iqbal, M. A. Reshchikov, L. He, and H. Morkoç, J. Electron. Mater. 32, 346 (2003)

${ }^{43}$ W. J. Moore, J. A. Freitas, Jr., and R. J. Molnar, Phys. Rev. B 56, 12073 (1997).

${ }^{44}$ L. Szaro, Surf. Sci. 137, 311 (1984).

${ }^{45}$ H. Dember, Z. Phys. 32, 554 (1931).

${ }^{46}$ A. Hierro, A. R. Arehart, B. Heying, M. Hansen, U. K. Mishra, S. P. DenBaars, J. S. Speck, and S. A. Ringel, Appl. Phys. Lett. 80, 805 (2002).

${ }^{47}$ B. I. Bednyi and N. V. Baidus, Semiconductors 27, 620 (1993).

${ }^{48}$ A. L. Musatov and S. Y. Smirnov, Surf. Sci. 269-270, 1048 (1992).

${ }^{49}$ M. Foussekis, A. A. Baski, and M. A. Reshchikov, in MRS Symposia Proceedings No. 1109 (Materials Research Society, Pittsburgh, 2009), p. B06-28.

${ }^{50}$ M. Foussekis, A. A. Baski, and M. A. Reshchikov, Appl. Phys. Lett. 94, 162116 (2009).

${ }^{51}$ T. A. Beierlein, W. Brütting, H. Riel, E. I. Haskal, P. Müller, and W. Rieß, Synth. Met. 111-112, 295 (2007).

${ }^{52}$ H. Nienhaus, M. Schneider, S. P. Grabowski, W. Mönch, R. Dimitrov, O. Ambacher, and M. Stutzmann, in MRS Symposia Proceedings No. 680 (Materials Research Society, Pittsburgh, 2001), p. E4.5.

${ }^{53}$ O. B. Aphek, L. Kronik, M. Leibovich, and Y. Shapira, Surf. Sci. 409, 485 (1998).

${ }^{54}$ G. Lucovsky, Solid State Commun. 3, 299 (1965).

${ }^{55}$ B. K. Ridley and M. A. Amano, J. Phys. C 14, 1255 (1981).

${ }^{56}$ During illumination with $580 \mathrm{~nm}$ light at $P_{0}=0.03 \mathrm{~W} / \mathrm{cm}^{2}$, only $\approx 10^{11}$ electrons are excited from one $\mathrm{cm}^{2}$ of the surface every second, in contrast to the $\approx 10^{15}$ holes reaching the surface every second under illumination with $365 \mathrm{~nm}$ light and the same power density. 